\title{
Survey of the Patterns of Using Stereotactic Ablative Radiotherapy for Early-Stage Non-small Cell Lung Cancer in Korea
}

\section{Sanghyuk Song, MD \\ Ji Hyun Chang, MD, PhD² \\ Hak Jae Kim, MD, $\mathrm{PhD}^{3}$ \\ Yeon Sil Kim, MD, PhD ${ }^{4}$ \\ Jin Hee Kim, MD, PhD \\ Yong Chan Ahn, MD, PhD' \\ Jae-Sung Kim, MD, PhD ${ }^{6}$ \\ Si Yeol Song, MD, $\mathrm{PhD}{ }^{7}$ \\ Sung Ho Moon, MD, PhD \\ Moon June Cho, MD, $\mathrm{PhD}^{9}$ \\ Seon Min Youn, MD, PhD ${ }^{10}$}

${ }^{*}$ A list author's affiliations appears at the end of the paper.

Correspondence: Hak Jae Kim, MD, PhD

Department of Radiation Oncology,

Seoul National University Hospital,

101 Daehak-ro, Jongno-gu, Seoul 03080, Korea

Tel: 82-2-2072-3177

Fax: 82-2-742-2073

E-mail: khjae@snu.ac.kr

Co-correspondence: Yeon Sil Kim, MD, PhD

Department of Radiation Oncology,

Seoul St. Mary's Hospital, College of Medicine,

The Catholic University of Korea,

222 Banpo-daero,

Seocho-gu, Seoul 06591, Korea

Tel: 82-2-2258-1501

Fax: 82-2-2258-1532

E-mail: yeonkim7@catholic.ac.kr

Received May 27, 2016

Accepted September 27, 2016

Published Online October 31, 2016

*Sanghyuk Song and Ji Hyun Chang contributed equally to this work.

\section{Purpose}

Stereotactic ablative radiotherapy (SABR) is an effective emerging technique for early-stage non-small cell lung cancer (NSCLC). We investigated the current practice of SABR for earlystage NSCLC in Korea.

\section{Materials and Methods}

We conducted a nationwide survey of SABR for NSCLC by sending e-mails to all board-certified members of the Korean Society for Radiation Oncology. The survey included 23 questions focusing on the technical aspects of SABR and 18 questions seeking the participants' opinions on specific clinical scenarios in the use of SABR for early-stage NSCLC. Overall, 79 radiation oncologists at 61/85 specialist hospitals in Korea (71.8\%) responded to the survey.

\section{Results}

SABR was used at 33 institutions (54\%) to treat NSCLC. Regarding technical aspects, the most common planning methods were the rotational intensity-modulated technique (59\%) and the static intensity-modulated technique (49\%). Respiratory motion was managed by gating (54\%) or abdominal compression (51\%), and $86 \%$ of the planning scans were obtained using 4-dimensional computed tomography. In the clinical scenarios, the most commonly chosen fractionation schedule for peripherally located T1 NSCLC was 60 Gy in four fractions. For centrally located tumors and T2 NSCLC, the oncologists tended to avoid SABR for radiotherapy, and extended the fractionation schedule.

\section{Conclusion}

The results of our survey indicated that SABR is increasingly being used to treat NSCLC in Korea. However, there were wide variations in the technical protocols and fractionation schedules of SABR for early-stage NSCLC among institutions. Standardization of SABR is necessary before implementing nationwide, multicenter, randomized studies.

\section{Key words}

Non-small cell lung carcinoma, Stereotactic body radiotherapy, Clinical practice pattern, Surveys and questionnaires 


\section{Introduction}

Surgical resection has long been the mainstay treatment option for early-stage non-small cell lung cancer (NSCLC). In recent decades, the introduction of stereotactic ablative radiotherapy (SABR) has provided an opportunity to cure NSCLC in patients with high surgical risks. SABR showed comparable local control with minimal toxicity compared with surgical resection in a retrospective study $[1,2]$. However, owing to its short history of development, there are still some controversies regarding the best strategies for SABR, including the optimal indication, dose, fractionation, planning method, and management of respiratory motion. In many countries, several studies have investigated the differences in the patterns of using SABR for NSCLC [1-3]. These differences in practice are potential hurdles to implementing multicenter trials. The purpose of the current study was to understand the current practices of using SABR for earlystage NSCLC in Korea. The lung cancer subcommittee of the Korean Society for Radiation Oncology intends to use these results to develop new protocols and standards for multicenter trials.

\section{Materials and Methods}

\section{Survey design}

This three-part survey was developed by the lung cancer subcommittee of the Korean Society for Radiation Oncology. SABR was defined as hypofractionated radiotherapy (1-8 fractions). The full questionnaire is provided in Supplementary. The first part of the survey recorded the responder and the institution characteristics, including the location; demographic characteristics of radiation oncologists, lung specialists, and medical physicists; how long SABR has been used to treat NSCLC; and the annual number of patients who undergo SABR for NSCLC. The second part of the survey focused on technical aspects of SABR for NSCLC, including the equipment used, planning systems, immobilization and motion-management methods, simulation techniques, image guidance, and quality assurance. The third part of the survey included questions about the clinical decisions and the choice of treatment modality for specific clinical scenarios. Some of the questions allowed multiple responses to reflect diverse opinions.

\section{Responses and statistics}

The survey was e-mailed to all board-certified members of the Korean Society for Radiation Oncology. The e-mail was sent four times between June 2014 and January 2015 to collect as many responses as possible. The survey results were collected and analyzed on March 2015. A total of 79 radiation oncologists responded, from 61 of the 85 institutions $(71.8 \%$ ) in South Korea that have a radiation oncology department.

Statistical analyses were performed using SPSS software (release 18.0.0, SPSS Inc., Chicago, IL). The $\chi^{2}$ test and Fisher exact test were used to identify differences among groups. A two-sided $p$-value of $\leq 0.05$ was considered statistically significant.

\section{Results}

\section{Characteristics of the institutions and tadiation oncolo- gists}

Of the 61 institutions that responded to survey, 33 used SABR for NSCLC and were evaluated in this study. There were 28 academic institutions (85\%) and five non-academic institutions (15\%). Nineteen institutions (58\%) were located in Seoul and its metropolitan areas. There were one or two board-certified radiation oncologists at each of 13 institutions $(40 \%)$, three at each of 12 institutions (36\%), and $\geq 3$ at each of eight institutions (24\%). Most institutions had one radiation oncologist who had specialized in lung cancer $(64 \%)$ together with one medical physicist (61\%). Eleven institutions $(33 \%)$ had used SABR to treat NSCLC for $<2$ years and $10(30 \%)$ had used SABR for $>5$ years. The time that SABR was first used to treat NSCLC is shown in Fig. 1. Overall, 37 radiation oncologists with experience of using SABR for lung cancer completed the survey and provided evaluable responses. Twenty-two of the respondents (59\%) worked in Seoul and its metropolitan areas. The characteristics of the radiation oncologists are shown in Table 1.

\section{Technical aspects and follow-up policy}

Multiple responses were allowed for questions on the technical aspects of SABR. Rotational intensity-modulation radiotherapy (IMRT) (59\%) was the most common planning method followed by static IMRT (49\%). Immobilization methods included the wing board and vacuum lock system, which were used by $54 \%$ and $51 \%$ of respondents, respectively. For motion management, $54 \%$ of the respondents used abdominal compression to control target movement and $51 \%$ 


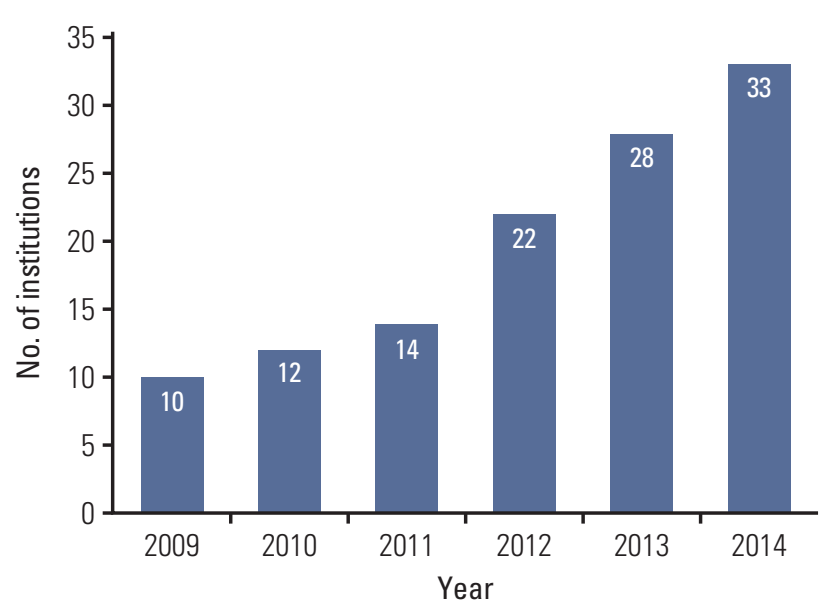

Fig. 1. Year in which stereotactic ablative radiotherapy was introduced at each institution.

Table 1. Characteristics of the radiation oncologists

\begin{tabular}{lr} 
Characteristic & No. of respondents (\%) \\
Academic affiliation & \\
Yes & $30(81)$ \\
No & $7(19)$ \\
Time since board certification (yr) & $3(8)$ \\
$<2$ & $7(19)$ \\
$2-5$ & $9(24)$ \\
$5-10$ & $18(49)$ \\
$>10$ & \\
How long the respondent & \\
has performed SABR (yr) & $16(43)$ \\
$<2$ & $11(30)$ \\
$2-5$ & $10(27)$ \\
$>5$ & \\
No. of cases of SABR per year & $14(38)$ \\
$<5$ & $9(24)$ \\
$5-10$ & $10(27)$ \\
$10-20$ & $4(11)$ \\
\hline 20 &
\end{tabular}

SABR, stereotactic ablative radiotherapy.

used respiratory gating. The technical aspects of SABR are summarized in Table 2 . With a fractionated schedule, $49 \%$ of respondents treat all patients on consecutive days, $38 \%$ used a more prolonged schedule, and 13\% used both schedules. Most of the respondents $(86 \%)$ obtained the first follow-up images at 4-7 weeks after SABR, and the most common imaging modalities were computed tomography (CT) (78\%),
Table 2. Technical aspects of stereotactic ablative radiotherapy

\begin{tabular}{|c|c|}
\hline Technical aspect & Respondent (\%) \\
\hline Four-dimensional CT simulation & 86 \\
\hline \multicolumn{2}{|l|}{ Planning system } \\
\hline Eclipse & 76 \\
\hline Pinnacle & 16 \\
\hline Accuray & 16 \\
\hline Other & 11 \\
\hline \multicolumn{2}{|l|}{ Planning method } \\
\hline Rotational IMRT & 59 \\
\hline Static IMRT & 49 \\
\hline Static non-coplanar beams & 35 \\
\hline CyberKnife & 19 \\
\hline Dynamic conformal arcs & 5 \\
\hline Protons & 5 \\
\hline Other & 8 \\
\hline Heterogeneity correction & 89 \\
\hline \multicolumn{2}{|l|}{ Motion management } \\
\hline Respiratory gating & 54 \\
\hline Abdominal compression & 51 \\
\hline Continuous implanted fiducial tracking & 11 \\
\hline Breath hold & 5 \\
\hline $\begin{array}{l}\text { Real-time electromagnetic } \\
\text { transponder tracking }\end{array}$ & 3 \\
\hline None & 8 \\
\hline \multicolumn{2}{|l|}{ Image guidance } \\
\hline $\mathrm{CT}$ & 94 \\
\hline Orthogonal kV image pair & 16 \\
\hline Electronic portal imaging & 11 \\
\hline Other & 14 \\
\hline
\end{tabular}

$\mathrm{CT}$, computed tomography; IMRT, intensity modulated radiotherapy.

followed by positron emission tomography (PET) (11\%), and both CT and PET (11\%).

\section{Clinical decisions}

The surveyed oncologists were asked whether they think it is necessary to obtain pathological confirmation before performing SABR in cases with a high risk of procedure complications or technical difficulties, if clinical T1 or T2 lung cancer is most likely. The oncologists frequently (46\%) recommended that patients should undergo pathologic confirmation by biopsy before performing SABR. The other options included that SABR could be performed only after CT or PET studies had been completed (38\%), and that SABR could be performed after a close imaging follow-up (14\%). The 

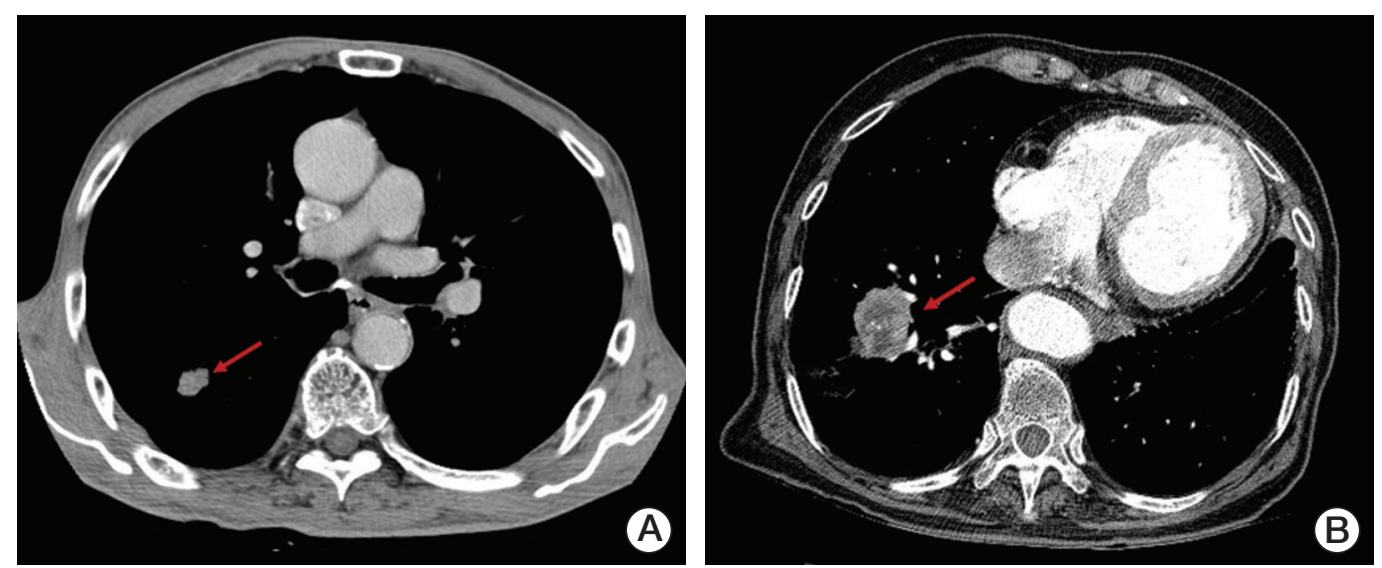

Fig. 2. (A) A 75-year-old male with peripherally located stage I, cT1 $(1.6 \mathrm{~cm}$ ), N0 non-small cell lung cancer (NSCLC) (arrow). (B) A 75-year-old male with medically inoperable stage I, cT2 $(4.5 \mathrm{~cm})$, N0 NSCLC (arrow).

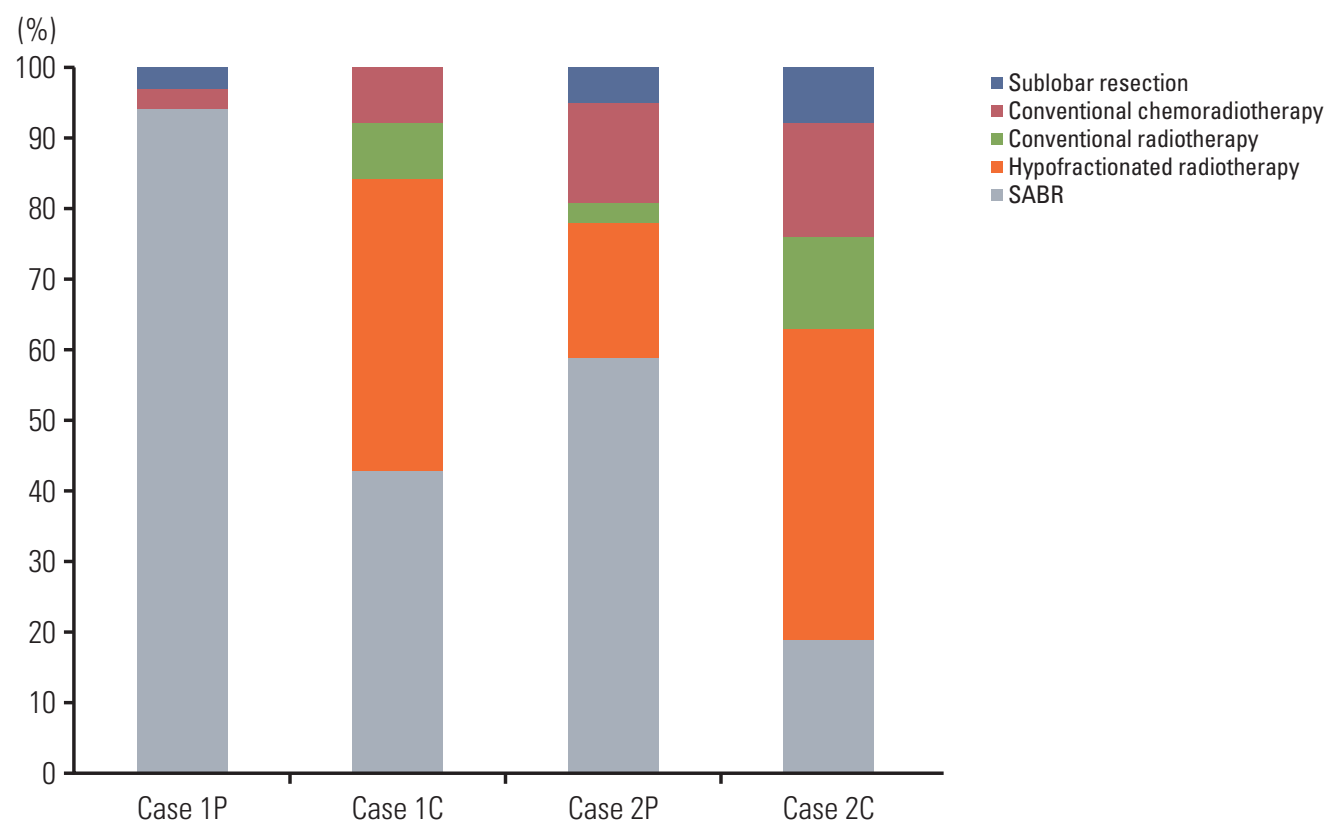

Fig. 3. Treatment options chosen for each case scenario. SABR, stereotactic ablative radiotherapy.

respondents were also asked how they would handle suspicious mediastinal or hilar lymph node enlargements diagnosed as reactive nodes by CT or PET. Fifty-four percent of the responders recommended pathologic confirmation before SABR, $41 \%$ recommended close observation with imaging modalities after SABR, 3\% responded that they would not perform SABR in this case, and one clinician would perform SABR in a T1 case and recommend patho- logic confirmation in a T2 case. We also asked the respondents to describe how pulmonary function test (PFT) results influence their decision to perform SABR. Most of the respondents $(62 \%)$ thought that the patient's clinical condition is more important than the PFT results, while $14 \%$ of the respondents stated that they would set limitations on PFT results which would not consider SABR. 


\section{Case management}

Case 1P was a 75-year-old male with stage I, cT1 $(1.6 \mathrm{~cm})$, N0 NSCLC located peripherally to the mediastinum and 2.2 $\mathrm{cm}$ from the chest wall (Fig. 2A). We assumed that he was diagnosed with adenocarcinoma, had good performance status (Zubrod 1), but his pulmonary function was too poor to perform lobectomy. Nearly all of the respondents $(94 \%)$ recommended SABR and the most frequently selected fractionation schedule was $60 \mathrm{~Gy}$ in four fractions $(46 \%)$ followed by $48 \mathrm{~Gy} /$ four fractions (19\%) and $60 \mathrm{~Gy} /$ three fractions (16\%).

Case $1 \mathrm{C}$ was identical to case $1 \mathrm{P}$, except the tumor was located $<2 \mathrm{~cm}$ from the mediastinum. For case $1 \mathrm{C}$, SABR was recommended by $43 \%$ of the respondents and hypofractionated conventional radiotherapy was recommended by $41 \%$. The most frequently selected fractionation schedule was 60 Gy in 10 fractions, chosen by $14 \%$ of respondents; however, there was little consensus with regard to the fractionation schedule. Other schedules used included $60 \mathrm{~Gy} / 15$ fractions $(11 \%)$ and $50 \mathrm{~Gy} /$ five fractions $(8 \%)$.

Case 2P was a 75-year-old male with stage I, cT2 $(4.5 \mathrm{~cm})$, N0 NSCLC with a performance status of Zubrod 1, but his pulmonary function was poor and he was scheduled to undergo lobectomy (Fig. 2B). Most of the respondents recommended radiotherapy alone (76\%). Concurrent chemoradiotherapy and sublobar resection were recommended by $14 \%$ and 5\%, respectively. SABR was selected as the treatment of choice by $59 \%$ of respondents and hypofractionated radiotherapy by $19 \%$ of respondents. The most frequently selected fractionation schedule was $60 \mathrm{~Gy}$ in four fractions $(32 \%)$.

Case $2 \mathrm{C}$ was identical to $2 \mathrm{P}$, except that it was central NSCLC $(<2 \mathrm{~cm}$ from the mediastinum). Radiotherapy alone, concurrent chemoradiotherapy, and sublobar resection were recommended by $76 \%, 16 \%$, and $8 \%$ of respondents, respectively. Only $19 \%$ of respondents considered SABR as the treatment of choice; most recommended hypofractionated conventional radiotherapy (44\%). The treatment options selected for each case are shown in Fig. 3.

\section{Discussion}

In this nationwide survey, we assessed the current practices of using SABR to treat early-stage NSCLC in Korea. SABR for NSCLC was rapidly incorporated into clinical practice in Korea (Fig. 1). Most of the institutions started SABR for NSCLC within the last 5 years. The majority of the radiation oncologists (72\%) with experience of SABR used this technique in $<10$ NSCLC patients per year. This low volume suggests that we should conduct multicenter trials rather than single-center trials in future studies of SABR for NSCLC.

The technical aspects of SABR varied widely among the institutions and could represent potential hurdles to multicenter trials. In particular, there were marked variations in the planning techniques and methods used to manage respiratory motion. Accordingly, it will be necessary to standardize these factors before commencing multicenter trials. Most institutions used modern imaging modalities with radiotherapy, including four-dimensional CT simulation, IMRT, and volumetric image guidance. These rapid adaptations of modern techniques make it feasible to perform high-quality trials.

Although SABR has been rapidly integrated into clinical practice, there are some issues to address. One of the issues is mandatory pathologic examination before treatment in high-risk patients. In our survey, $46 \%$ of the respondents sought pathologic confirmation before performing SABR. Another issue is the value of mediastinal staging. More than half of the respondents stated that pathologic confirmation was necessary before SABR in cases with reactive mediastinal nodes. Of course, extensive diagnostic staging procedures will be required in multicenter trials.

Currently, various fractionation schedules are used for SABR, including $20 \mathrm{~Gy} \times 3,18 \mathrm{~Gy} \times 3,16 \mathrm{~Gy} \times 3,15 \mathrm{~Gy} \times 4,12$ Gy $\times 4$, and 12 Gy $\times 5$ fractions [4]. To compare the efficacy or toxicity of two different dose fractionation schemes, we conventionally use the biologically effective dose (BED), which is calculated from a linear quadratic model. It has also been adopted in studies comparing SABR fractionation schemes. It has been widely debated whether we should apply a LQ model to SABR, but multiple studies have revealed that radiotherapy doses exceeding a $\mathrm{BED}_{10}$ of $100 \mathrm{~Gy}$ or $105 \mathrm{~Gy}$ increase the local control rate and survival outcome, which suggests that the LQ model is suitable for comparing conventional dose schemes [5-9]. However, there is no consensus regarding the ideal fractionation schedule for SABR of NSCLC. In the present survey, even in cases with peripheral CT1 NSCLC, the most popular fractionation regimen (60 Gy in four fractions) was used by less than half of the respondents. This might be due to the national health insurance program of South Korea, which covers SABR only when SABR is given in four fractions. In T2 or central cases, the fractionation schedule varied considerably. Accordingly, it will be necessary to standardize the dose and fraction schedule for SABR before starting future trials.

We investigated the opinions of radiation oncologists regarding their use of SABR in cases with centrally located and/ or large (cT2) cancers. Results of a phase II trial performed at the University of Indiana revealed a trend toward increased grade 3-5 toxicity in central tumors (27.3\% vs. 
$10.4 \%, \mathrm{p}=0.088)[10]$, raising concern of increased toxicity of SABR for centrally located NSCLC. Accordingly, fewer respondents recommended SABR for central NSCLC than for peripheral NSCLC in the current survey (Fig. 3). In terms of tumor size, T2 NSCLC was associated with a lower local control rate than T1 NSCLC following SABR in prior studies $[11,12]$. The part of the survey focusing on preferred treatments for T2 NSCLC revealed that concurrent chemotherapy was more common in T1 cases than in T2 cases. Many radiation oncologists hesitated to use SABR for centrally located T2 NSCLC. Only 19\% of the respondents stated that they would perform SABR in cases with centrally located T2 NSCLC. Because of the varying influences of location and size of the cancer on clinical practices, clinical trials should include stratified protocols to account for these factors. Our study has several limitations. First, not all of the board-certified radiation oncologists in Korea responded to the survey, which might introduce selection bias. In addition, because multiple responses were allowed for some of the technical questions, we may overestimate the rate of infrequently used techniques. Finally, although we sought to integrate most of the approaches used in actual practice, some practices were not reported because the list of responses was incomplete.

\section{Conclusion}

In conclusion, this nationwide survey confirmed that the use of SABR for treating early-stage NSCLC has increased dramatically in Korea in recent years. The technical aspects and clinical decisions for SABR for treating NSCLC varied markedly. The treatment patterns of centrally located T2 NSCLC varied much more than those of peripherally located T1 NSCLC. These results highlight the need to standardize various aspects of the protocol when planning nationwide, multicenter, clinical trials.

\section{Electronic Supplementary Material}

Supplementary materials are available at Cancer Research and Treatment website (http:// www.e-crt.org).

\section{Conflicts of Interest}

Conflict of interest relevant to this article was not reported.

\section{Author Details}

${ }^{1}$ Department of Radiation Oncology, Samsung Medical Center, Seoul, ${ }^{2}$ Department of Radiation Oncology, SMG-SNU Boramae Medical Center, Seoul, ${ }^{3}$ Department of Radiation Oncology, Seoul National University Hospital, Seoul, ${ }^{4}$ Department of Radiation Oncology, Seoul St. Mary's Hospital, Seoul, ${ }^{5}$ Department of Radiation Oncology, Keimyung University Hospital, Daegu, ${ }^{6}$ Department of Radiation Oncology, Seoul National University Bundang Hospital, Seongnam, ${ }^{7}$ Department of Radiation Oncology, Asan Medical Center, Seoul, ${ }^{8}$ Proton Therapy Center, National Cancer Center, Goyang, ' ${ }^{2}$ Department of Radiation Oncology, Chungnam National University Hospital, Daejeon, ${ }^{10}$ Department of Radiation Oncology, Eulji University Hospital, Daejeon, Korea

\section{References}

1. Corso CD, Park HS, Moreno AC, Kim AW, Yu JB, Husain ZA, et al. Stage I lung SBRT clinical practice patterns. Am J Clin Oncol. 2014 Dec 10 [Epub]. https://doi.org/10.1097/COC. 0000000000000162.

2. Kong FM, Cuneo KC, Wang L, Bonner JA, Gaspar LE, Komaki $\mathrm{R}$, et al. Patterns of practice in radiation therapy for non-small cell lung cancer among members of the American Society for Radiation Oncology. Pract Radiat Oncol. 2014;4:e133-41.
3. Guckenberger M, Allgauer M, Appold S, Dieckmann K, Ernst I, Ganswindt U, et al. Safety and efficacy of stereotactic body radiotherapy for stage 1 non-small-cell lung cancer in routine clinical practice: a patterns-of-care and outcome analysis. J Thorac Oncol. 2013;8:1050-8.

4. Koshy M, Malik R, Weichselbaum RR, Sher DJ. Increasing radiation therapy dose is associated with improved survival in patients undergoing stereotactic body radiation therapy for 
stage I non-small-cell lung cancer. Int J Radiat Oncol Biol Phys. 2015;91:344-50.

5. Rowe BP, Boffa DJ, Wilson LD, Kim AW, Detterbeck FC, Decker RH. Stereotactic body radiotherapy for central lung tumors. J Thorac Oncol. 2012;7:1394-9.

6. Onishi H, Araki T, Shirato H, Nagata Y, Hiraoka M, Gomi K, et al. Stereotactic hypofractionated high-dose irradiation for stage I nonsmall cell lung carcinoma: clinical outcomes in 245 subjects in a Japanese multiinstitutional study. Cancer. 2004;101:1623-31.

7. Grills IS, Hope AJ, Guckenberger M, Kestin LL, Werner-Wasik M, Yan D, et al. A collaborative analysis of stereotactic lung radiotherapy outcomes for early-stage non-small-cell lung cancer using daily online cone-beam computed tomography image-guided radiotherapy. J Thorac Oncol. 2012;7:1382-93.

8. Olsen JR, Robinson CG, El Naqa I, Creach KM, Drzymala RE, Bloch $\mathrm{C}$, et al. Dose-response for stereotactic body radiotherapy in early-stage non-small-cell lung cancer. Int J Radiat Oncol Biol Phys. 2011;81:e299-303.
9. Chang JY, Balter PA, Dong L, Yang Q, Liao Z, Jeter M, et al. Stereotactic body radiation therapy in centrally and superiorly located stage I or isolated recurrent non-small-cell lung cancer. Int J Radiat Oncol Biol Phys. 2008;72:967-71.

10. Fakiris AJ, McGarry RC, Yiannoutsos CT, Papiez L, Williams M, Henderson MA, et al. Stereotactic body radiation therapy for early-stage non-small-cell lung carcinoma: four-year results of a prospective phase II study. Int J Radiat Oncol Biol Phys. 2009;75:677-82.

11. Dunlap NE, Larner JM, Read PW, Kozower BD, Lau CL, Sheng K, et al. Size matters: a comparison of T1 and T2 peripheral non-small-cell lung cancers treated with stereotactic body radiation therapy (SBRT). J Thorac Cardiovasc Surg. 2010;140: 583-9.

12. Bral S, Gevaert T, Linthout N, Versmessen H, Collen C, Engels $\mathrm{B}$, et al. Prospective, risk-adapted strategy of stereotactic body radiotherapy for early-stage non-small-cell lung cancer: results of a Phase II trial. Int J Radiat Oncol Biol Phys. 2011;80:1343-9. 